\title{
Fatores relacionados à eficiência da propulsáo em cadeira de rodas manual de usuários com paraplegia devido à lesáo medular
}

\author{
Factors related to propulsion efficiency in manual wheelchair users with \\ paraplegia due to spinal cord injury
}

\author{
Haidar Tafner Curi ${ }^{\mathrm{a}}$ (D), Jaqueline de Lima ${ }^{\mathrm{b}}$ (D), Eliana Chaves Ferretti ${ }^{\mathrm{a}}$ (D) \\ ${ }^{a}$ Universidade Federal de São Paulo - UNIFESP, Campus Baixada Santista, Santos, SP, Brasil. \\ ${ }^{b}$ Centro Especializado em Reabilitação - CER, Santos, SP, Brasil.
}

Como citar: Curi, H. T., Lima, J., \& Ferretti, E. C. (2020). Fatores relacionados à eficiência da propulsão em cadeira de rodas manual de usuários com paraplegia devido à lesão medular. Cadernos Brasileiros de Terapia Ocupacional. 28(3), 999-1019. https://doi.org/10.4322/2526-8910.ctoAR1935

\begin{abstract}
$\underline{\text { Resumo }}$
Introdução: Indivíduos com paraplegia devido à lesão medular (LM) realizam propulsão de cadeira de rodas manual (CRM), a fim de promover mobilidade funcional para realização das atividades cotidianas. No entanto, a ineficiência da propulsão causada pela inadequada configuração da CRM, assim como dores e lesôes nos membros superiores (MMSS), pode resultar na diminuição da mobilidade do usuário. Objetivo: Por meio da metodologia da revisão integrativa, buscou-se identificar e avaliar fatores relacionados à eficiência da propulsão em CRM de usuários com paraplegia devido à LM. Método: Foram selecionados estudos indexados na PubMed, LILACS e SciELO sobre a biomecânica da propulsão de usuários de CRM com paraplegia devido à LM, entre os anos de 2008 e 2018. Resultados: Dentre os 10 estudos incluídos na revisão, dois foram classificados como nível III-2 e oito como nível IV de evidência. Foram identificados como fatores relacionados à eficiência da propulsão: os momentos não propulsivos; a velocidade dos MMSS na fase de recuperação; a posição da mão no período de liberação; o tamanho do encosto; a manutenção do peso corporal; o nível de atividade diária e a força de adutores de ombro; a intensidade da propulsáo; a orientação dos MMSS; e o tempo de LM. Conclusáo: As evidências sobre ciclo e padrōes de propulsão, configurações de CRM, características do usuário e dores e lesões nos MMSS demonstraram ser fatores relacionados à eficiência da propulsão em CRM de usuários com paraplegia devido à LM.
\end{abstract}

Palavras-chave: Cadeira de Rodas, Paraplegia, Traumatismos da Medula Espinal, Biomecânica. 


\begin{abstract}
$\underline{\text { Abstract }}$
Introduction: Individuals with paraplegia due to spinal cord injury (SCI) perform manual wheelchair (MWC) propulsion to promote functional mobility to perform daily activities. However, the inefficiency of propulsion caused by inadequate MWC setting, as well as pain and upper limb injury (UL), can result in decreased user mobility. Objective: Through the methodology of the integrative review, sought to identify and evaluate factors related to propulsion efficiency in MWC of users with paraplegia due to SCI. Method: We selected indexed studies in PubMed, LILACS, and SciELO on the biomechanics of the propulsion of users with paraplegia due to SCI between 2008 and 2018. Results: Among the 10 studies included in the review, two studies were classified as level III-2 and eight as level IV evidence. Factors related to propulsion efficiency were identified as: non-propulsive moments; the speed of the UL in the recovery phase; the position of the hand in the release period; the size of the backrest; maintaining body weight; the level of daily activity and shoulder adduction strength; the intensity of the propulsion; the orientation of the UL and the SCI time. Conclusion: Evidence regarding the cycle and propulsion patterns, MWC settings, user characteristics, and pain and injury in the UL proved to be factors related to propulsion efficiency in MWC of users with SCI paraplegia.
\end{abstract}

Keywords: Wheelchair, Paraplegia, Spinal Cord Injuries, Biomechanics.

\title{
1 Introdução
}

A lesão medular (LM) é caracterizada por danos nas estruturas do canal medular, resultando em implicações físicas, psicológicas e sociais (Polia \& Castro, 2007). De acordo com a World Health Organization (2013), a incidência mundial de LM é de 40 a 80 casos por milhão de habitantes. As causas mais comuns são por acidentes de trânsito, quedas, violências e lesão por esportes. No Brasil, a incidência corresponde a 71 casos/1 milhão de habitantes por ano (Masini, 2001). Estima-se que há mais de 180 mil casos de LM, com aumento de aproximadamente 10 mil casos por ano (Masini, 2001).

Indivíduos com paraplegia devido à LM comumente realizam propulsão de cadeira de rodas manual (CRM), a fim de promover mobilidade para realização das atividades cotidianas (Cooper et al., 1998). No entanto, a ineficiência da propulsão causada pela inadequada configuração da CRM (Medola et al., 2014), assim como dores e lesóes nos membros superiores (MMSS) (Requejo et al., 2008), pode resultar na diminuição da mobilidade do usuário.

Diversos estudos investigaram fatores relacionados à eficiência da propulsão em CRM. Esses estudos descrevem que as cadeiras de rodas ultralight, constituídas de fibra de carbono e titanium, são mais leves e resistentes (Cooper et al., 1997) e, consequentemente, exigem menos esforços e movimentos repetitivos realizados pelo usuário durante a propulsão, evitando o risco de dores e lesôes nos MMSS (Requejo et al., 2008; Chow \& Levy, 2011). Assim como a estrutura da CRM, o uso de rodas traseiras produzidas com fibra de carbono contribui para preservar a função dos 
MMSS, em razão de serem mais leves e minimizarem a transmissão de impactos ao corpo do usuário (DiGiovine et al., 2006). As rodas dianteiras também requerem atenção quanto ao seu diâmetro e material de produção. $\mathrm{O}$ uso de rodas dianteiras com menor diâmetro e do tipo pneumáticas ou fibra de carbono favorece para diminuir a resistência ao rolamento, além de minimizar os impactos ao MMSS (Kwarciak et al., 2009b; Medola et al., 2014).

Estudos de revisão da literatura demonstram que dores e lesóes no ombro (Requejo et al., 2008; Chow \& Levy, 2011) e a configuração dos componentes da cadeira de rodas (Medola et al., 2014) são fatores relacionados à propulsão manual. Compreender esses fatores é essencial para promover aumento da mobilidade e participação do usuário e, portanto, melhora da qualidade de vida (Chow \& Levy, 2011). Pessoas com paraplegia utilizam os MMSS para realizar propulsão manual e, consequentemente, ocorre aumento da demanda dessas estruturas (Requejo et al., 2008; Chow \& Levy, 2011). Essa condição pode aumentar o risco de dores e lesôes no ombro, limitando o desempenho do usuário durante as atividades de vida diária (Requejo et al., 2008; Chow \& Levy, 2011). Ainda, estudos demonstram que o ajuste inadequado da altura do encosto, da cambagem e do eixo horizontal/vertical das rodas traseiras pode afetar a amplitude de movimento das articulaçôes dos MMSS e a estabilidade do usuário durante a propulsão manual. A configuração inadequada desses componentes pode afetar a eficiência da propulsão, limitando o desempenho da mobilidade do usuário (Medola et al., 2014).

Apesar da contribuição dos artigos de revisão presentes na literatura científica (Requejo et al., 2008; Chow \& Levy, 2011; Medola et al., 2014), novas evidências foram fornecidas por estudos recentes que investigaram fatores relacionados à eficiência da propulsão. Sendo assim, o presente artigo de revisão, além de apresentar e discutir novas evidências científicas, especificamente direcionadas aos usuários de CRM com paraplegia devido à LM, avalia e classifica os estudos de acordo com os níveis da evidência científica, utilizando-se da metodologia da revisão integrativa.

A Prática Baseada em Evidência (PBE) é uma abordagem que busca identificar e avaliar as evidências científicas para aplicabilidade na prática clínica, considerando a experiência do profissional e a opinião do cliente no processo de tomada de decisão (Souza et al., 2010). A revisão integrativa é um método da PBE, que possibilita incluir estudos experimentais e não-experimentais; descrever conceitos; identificar, analisar e sintetizar os resultados de uma temática específica, proporcionando ao profissional de saúde a escolha da melhor evidência possível no cuidado do paciente/cliente/usuário (Souza et al., 2010).

O terapeuta ocupacional é o profissional habilitado para prescrever dispositivos assistivos de mobilidade; desenvolver adaptações e estratégias para auxiliar nas AVD (atividades de vida diária) e AIVD (atividades instrumentais de vida diária); e orientar, treinar e acompanhar os usuários e familiares quanto ao uso do dispositivo assistivo (Brasil, 2015). Logo, este artigo também contribuirá com os terapeutas ocupacionais que atuam na prática e em serviços de dispensação de cadeira de rodas, utilizando-se das evidências dos estudos selecionados, do pensamento crítico profissional e considerando a opinião dos usuários, a fim de promover propulsão em CRM com eficiência. Portanto, por meio da metodologia da revisão integrativa, o presente estudo tem como objetivo 
identificar e avaliar os fatores relacionados à eficiência da propulsão em CRM de usuários com paraplegia devido à LM.

\section{Método}

O presente estudo incorporou a metodologia da revisão integrativa, com o propósito de analisar o conhecimento de diversos estudos, classificando-os de acordo com os níveis da evidência científica para eventual aplicabilidade dos resultados na prática clínica (Souza et al., 2010). As etapas da revisão integrativa são compreendidas em: 1) elaboração da questáo norteadora da pesquisa; 2) busca de estudos nas bases de dados e estabelecimento dos critérios de inclusão/exclusão da amostra; 3) coleta de dados dos estudos selecionados; 4) análise crítica dos estudos e classificação por níveis de evidência; 5) discussão dos resultados; 6) apresentação da revisão integrativa (Souza et al., 2010).

Sendo assim, o estudo foi desenvolvido com base na seguinte questão norteadora: quais fatores estão relacionados à eficiência da propulsão em CRM de usuários com paraplegia devido à LM? Para tal fim, foi realizada uma busca de estudos nas fontes de indexação PubMed, LILACS e SciELO, na língua inglesa e portuguesa, entre os anos de 2008 e 2018, utilizando descritores de acordo com o DeCS (Descritores em Ciências da Saúde): wheelchairs (cadeira de rodas), spinal cord injuries (traumatismos da medula espinal) e paraplegia (paraplegia). Utilizaram-se ainda outros termos significativos que não estão presentes no DeCS, como: propulsion (propulsão) e biomechanics (biomecânica). Além da busca de estudos nas bases de dados, realizou-se uma busca manual na lista de referência de todos os artigos que foram selecionados para a leitura na íntegra.

Como critérios de inclusão, no processo de busca foram considerados: estudos referentes à biomecânica da propulsão em CRM; estudos que envolvem usuários de CRM (homens e mulheres) com paraplegia devido à LM; e artigos de periódicos publicados nos idiomas português e inglês. Como critérios de exclusão, não foram considerados: estudos envolvendo handciclyng e cadeiras de rodas motorizadas; artigos editoriais; estudos de simulação; estudos piloto; revisões narrativas; estudos de caso qualitativo; relatos de caso; e estudos com resultados de grupos constituídos por apenas indivíduos tetraplégicos e/ou com outros tipos de doenças/condiçóes de saúde.

Os dados coletados nos estudos selecionados foram sistematizados na tabela de evidências e categorizados por temas para a discussão dos resultados. Todos os estudos foram avaliados criteriosamente, baseando-se nos níveis da evidência científica fornecidos por National Health and Medical Research Council (1998). A classificação da evidência científica é determinada de acordo com o desenho de estudo utilizado, sendo organizada hierarquicamente em 4 níveis: I) evidências obtidas com base em uma revisão sistemática de ensaios clínicos randomizados; II) evidências obtidas com base em ensaio clínico randomizado; III-1) evidências obtidas de ensaios controlados pseudorandomizados; III-2) evidências obtidas de estudos comparativos com controles e alocação não randomizada, estudos de coorte, estudos caso-controle ou série temporal interrompida com grupo controle; III-3) evidências obtidas de estudos comparativos com controle histórico, dois ou mais estudos de grupo único ou série temporal interrompida sem um grupo controle paralelo; IV) evidências obtidas com base em séries 
de casos. As evidências dos artigos incluídos na revisão foram discutidas com os demais artigos presentes na literatura científica referente à temática (Souza et al., 2010).

\section{Resultados}

No total, foram encontrados 68 estudos (PubMed n=35; LILACS n=23; SciELO $\mathrm{n}=10$ ), sendo que, desses, 10 foram excluídos por duplicata. Dentre os artigos que foram selecionados para leitura do título e resumo $(\mathrm{n}=58), 23$ foram excluídos. Após a leitura na íntegra dos estudos selecionados $(\mathrm{n}=35), 27$ foram excluídos. Por fim, com base na lista de referências dos artigos selecionados para a leitura na íntegra, foram selecionados dois artigos (Mulroy et al., 2015; Gil-Agudo et al., 2014) por busca manual nos artigos de Requejo et al. (2015) e Gil-Agudo et al. (2014), respectivamente, totalizando 10 estudos incluídos na revisão (Figura 1).

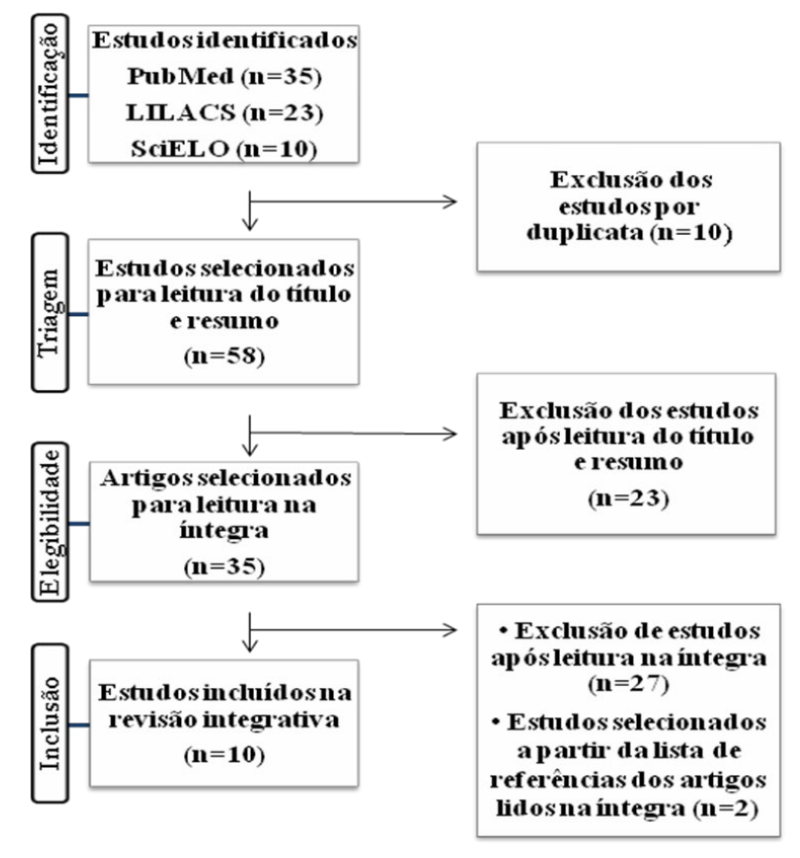

Figura 1. Fluxograma de seleção dos estudos. Fonte: Elaborada pelo autor.

Os estudos selecionados apresentaram as seguintes temáticas: ciclo e padróes de propulsão, configuraçooes da CRM, características do usuário de CRM e dores e lesões nos MMSS. As evidências classificadas com maior nível foram fornecidas por Mulroy et al. (2015) e Gil-Agudo et al. (2015). Por outro lado, os demais estudos foram classificados como nível IV de evidência (Tabela 1).

A maioria dos estudos englobou indivíduos adultos de ambos os sexos, exceto os estudos de Gil-Agudo et al. (2014, 2015), que apresentaram amostras constituídas apenas por homens. Somente o estudo de Raina et al. (2012) não informou a idade média dos participantes. Os estudos foram originados nos Estados Unidos $(n=7)$, Espanha $(n=2)$ e Taiwan $(n=1)$. 
Tabela 1. Síntese dos estudos classificados por níveis de evidências.

\begin{tabular}{|c|c|c|c|c|c|}
\hline Autor & $\begin{array}{l}\text { Nível de } \\
\text { evidência }\end{array}$ & Objetivo & Participantes & $\begin{array}{c}\text { Coleta de } \\
\text { dados }\end{array}$ & Resultados \\
\hline $\begin{array}{c}\text { Kwarciak et al. } \\
\text { (2009a) /EUA }\end{array}$ & IV & $\begin{array}{c}\text { Criar uma } \\
\text { definição } \\
\text { abrangente do } \\
\text { ciclo da } \\
\text { propulsão em } \\
\text { CRM e } \\
\text { demonstrar o } \\
\text { seu benefício } \\
\text { clínico. }\end{array}$ & $\begin{array}{c}\mathrm{n}=54 \\
\text { (44 homens e } \\
10 \text { mulheres); } \\
\text { Tempo médio } \\
\text { de lesão: } \\
14,4 \text { anos } \\
( \pm 10,4) ; \\
\text { Idade média: } \\
40,7 \text { anos } \\
( \pm 11,3) ; \\
\text { Peso corporal: } \\
76,6 \text { kg }( \pm 16.4)\end{array}$ & $\begin{array}{c}\text { Dados cinéticos } \\
\text { e cinemáticos } \\
\text { foram coletados } \\
\text { durante a } \\
\text { propulsão de } \\
\text { CRM no } \\
\text { dinamômetro. }\end{array}$ & $\begin{array}{c}\text { O ciclo da } \\
\text { propulsão foi } \\
\text { dividido em: } \\
\text { fase de contato } \\
\text { (contato inicial, } \\
\text { propulsáo e } \\
\text { liberaçáo) e fase } \\
\text { de recuperação; } \\
\text { Os períodos de } \\
\text { contato inicial } \\
\text { e liberaçáa } \\
\text { foram } \\
\text { considerados } \\
\text { como } \\
\text { momentos não } \\
\text { propulsivos do } \\
\text { contato da mão } \\
\text { com o aro; } \\
\text { A perda de } \\
\text { potência e o } \\
\text { aumento do } \\
\text { momento de } \\
\text { frenagem } \\
\text { ocorre } \\
\text { conforme a } \\
\text { velocidade de } \\
\text { impulso } \\
\text { aumenta } \\
\text { durante os } \\
\text { períodos de } \\
\text { contato inicial } \\
\text { e de liberação; } \\
\text { O uso de um } \\
\text { padrão de } \\
\text { propulsão com } \\
\text { as mãos abaixo } \\
\text { do aro durante } \\
\text { o período de } \\
\text { contato inicial } \\
\text { diminuiu o } \\
\text { momento de } \\
\text { frenagem e } \\
\text { aumentou a } \\
\text { potência. }\end{array}$ \\
\hline
\end{tabular}


Tabela 1. Continuação...

\begin{tabular}{|c|c|c|c|c|c|}
\hline Autor & $\begin{array}{l}\text { Nível de } \\
\text { evidência }\end{array}$ & Objetivo & Participantes & $\begin{array}{c}\text { Coleta de } \\
\text { dados }\end{array}$ & Resultados \\
\hline $\begin{array}{l}\text { Raina et al. } \\
\text { (2012) /EUA }\end{array}$ & IV & $\begin{array}{l}\text { Determinar se a } \\
\text { magnitude da } \\
\text { velocidade da } \\
\text { mão/antebraço e } \\
\text { a força de reaçáo } \\
\text { dependem da } \\
\text { técnica de } \\
\text { propulsão. }\end{array}$ & $\begin{array}{c}\mathrm{n}=34 \\
\text { (31 homens e } \\
3 \text { mulheres); } \\
\text { Tempo médio } \\
\text { de lesão: } S D ; \\
\text { Idade média: } \\
\text { SD; } \\
\text { Peso corporal } \\
\text { médio: } \\
74,5 \text { anos }( \pm 12)\end{array}$ & $\begin{array}{c}\text { Dados cinéticos } \\
\text { e cinemáticos } \\
\text { foram coletados } \\
\text { durante a } \\
\text { propulsão da } \\
\text { CRM no } \\
\text { ergômetro } \\
\text { estacionário. }\end{array}$ & $\begin{array}{c}\text { Os participantes } \\
\text { com paraplegia } \\
\text { utilizaram, } \\
\text { preferencialment } \\
\text { e, o padrão } \\
\text { SLOP em } \\
\text { comparação a } \\
\text { outros padróes } \\
\text { (ARC e SC); } \\
\text { A velocidade da } \\
\text { máo/antebraço } \\
\text { antes do contato } \\
\text { com o aro foi } \\
\text { correlacionada } \\
\text { com a força } \\
\text { efetiva durante a } \\
\text { propulsão; } \\
\text { Não houve } \\
\text { correlação entre } \\
\text { a força de reação } \\
\text { e os padrões de } \\
\text { propulsão. }\end{array}$ \\
\hline $\begin{array}{l}\text { Requejo et al. } \\
(2015) \text { /EUA }\end{array}$ & IV & $\begin{array}{l}\text { Investigar as } \\
\text { relações entre a } \\
\text { localização da } \\
\text { mão nos } \\
\text { períodos de } \\
\text { contato inicial e } \\
\text { de liberação, e } \\
\text { características de } \\
\text { propulsão, } \\
\text { forças de reação } \\
\text { e cinética do } \\
\text { ombro durante a } \\
\text { propulsão. }\end{array}$ & $\begin{array}{c}\mathrm{n}=222 \\
\text { (198 homens e } \\
24 \text { mulheres); } \\
\text { Tempo médio } \\
\text { de lesão: } \\
9,3 \text { anos }( \pm 6.1) \text {; } \\
\text { Idade média: } \\
34,7 \text { anos } \\
( \pm 9,3) ; \\
\text { Peso corporal } \\
\text { médio: } 74,4 \mathrm{~kg} \\
( \pm 15,9)\end{array}$ & $\begin{array}{c}\text { Foram coletados } \\
\text { dados cinéticos e } \\
\text { cinemáticos } \\
\text { durante a } \\
\text { propulsáo de } \\
\text { CRM no } \\
\text { ergômetro } \\
\text { estacionário. }\end{array}$ & $\begin{array}{l}\text { O ACP e o ALA } \\
\text { da mão } \\
\text { proporcionaram } \\
\text { cadência } \\
\text { reduzida e maior } \\
\text { distância entre os } \\
\text { ciclos. Somente } \\
\text { o ALA foi } \\
\text { associado com o } \\
\text { aumento da } \\
\text { velocidade; } \\
\text { O ALA produziu } \\
\text { aumento de } \\
\text { forças no aro e } \\
\text { sobre o ombro } \\
\text { bem como nos } \\
\text { momentos de } \\
\text { adutores e } \\
\text { rotadores } \\
\text { externos; } \\
\text { O ACP produziu } \\
\text { aumento da } \\
\text { força posterior e } \\
\text { de momentos } \\
\text { flexores de } \\
\text { ombro. }\end{array}$ \\
\hline
\end{tabular}


Tabela 1. Continuação...

\begin{tabular}{|c|c|c|c|c|c|}
\hline Autor & $\begin{array}{l}\text { Nível de } \\
\text { evidência }\end{array}$ & Objetivo & Participantes & $\begin{array}{l}\text { Coleta de } \\
\text { dados }\end{array}$ & Resultados \\
\hline $\begin{array}{c}\text { Yang et al. } \\
\text { (2012) } \\
\text { /Twaian }\end{array}$ & IV & $\begin{array}{c}\text { Avaliar o efeito } \\
\text { da altura do } \\
\text { encosto sobre } \\
\text { a cinemática e } \\
\text { cinética } \\
\text { durante a } \\
\text { propulsão. }\end{array}$ & $\begin{array}{c}\mathrm{n}=(26 \text { homens } \\
\mathrm{e} \\
10 \text { mulheres }) \text {; } \\
\text { Tempo médio } \\
\text { de lesão: } \\
11,88 \text { anos } \\
( \pm 8,4) ; \\
\text { Idade média: } \\
39,1 \text { anos } \\
\text { ( } \pm 10,5) ; \\
\text { Peso corporal } \\
\text { médio: } \mathrm{SD}\end{array}$ & $\begin{array}{c}\text { Dados } \\
\text { cinéticos e } \\
\text { cinemáticos } \\
\text { foram } \\
\text { coletados } \\
\text { durante a } \\
\text { propulsão na } \\
\text { CRM sobre a } \\
\text { esteira } \\
\text { motorizada } \\
\text { na condiçãoo } \\
\text { nivelada ou } \\
\text { inclinada } \\
\text { (3o), } \\
\text { utilizando um } \\
\text { encosto baixo } \\
\text { ou alto. }\end{array}$ & $\begin{array}{c}\text { O uso do } \\
\text { encosto baixo } \\
\text { permitiu } \\
\text { maior ADM } \\
\text { do ombro, } \\
\text { cadência } \\
\text { reduzida e } \\
\text { aumento do } \\
\text { ângulo e do } \\
\text { tempo de } \\
\text { impulso, } \\
\text { independente } \\
\text { do } \\
\text { nivelamento } \\
\text { ou inclinaçấo } \\
\text { da esteira. }\end{array}$ \\
\hline $\begin{array}{l}\text { Collinger et al } \\
\text {. (2008) /EUA }\end{array}$ & IV & $\begin{array}{c}\text { Apresentar } \\
\text { uma análise } \\
\text { descritiva e } \\
\text { comparativa } \\
\text { da cinética e } \\
\text { cinemática do } \\
\text { ombro durante } \\
\text { a propulsão } \\
\text { em CRM. }\end{array}$ & $\begin{array}{c}\mathrm{n}=61 \\
\text { (49 homens e } \\
12 \text { mulheres); } \\
\text { Tempo médio } \\
\text { de lesão: } \\
\text { 14,6 anos } \\
( \pm 10,5) ; \\
\text { Idade média: } \\
43,1 \text { anos } \\
( \pm 12) ; \\
\text { Peso corporal } \\
\text { médio: } 75,9 \mathrm{~kg} \\
( \pm 14)\end{array}$ & $\begin{array}{c}\text { Dados } \\
\text { cinéticos e } \\
\text { cinemáticos } \\
\text { foram } \\
\text { coletados } \\
\text { durante a } \\
\text { propulsáo na } \\
\text { CRM sobre o } \\
\text { dinamômetro } \\
\text { em diferentes } \\
\text { velocidades } \\
\text { (auto } \\
\text { selecionada; } \\
0,9 \mathrm{~m} / \mathrm{s}, \\
1,8 \mathrm{~m} / \mathrm{s} \text { ). }\end{array}$ & $\begin{array}{c}46 \% \text { dos } \\
\text { participantes } \\
\text { relataram } \\
\text { experiências } \\
\text { de dores no } \\
\text { ombro } \\
\text { anteriormente } \\
\text { ao estudo; } \\
\text { O peso dos } \\
\text { participantes } \\
\text { foi o principal } \\
\text { fator para } \\
\text { aumentar as } \\
\text { forças e } \\
\text { momentos de } \\
\text { ombro } \\
\text { durante a } \\
\text { propulsão; } \\
\text { As forças e } \\
\text { momentos de } \\
\text { ombro } \\
\text { aumentaram } \\
\text { conforme o } \\
\text { aumento da } \\
\text { velocidade. }\end{array}$ \\
\hline
\end{tabular}


Tabela 1. Continuação...

\begin{tabular}{|c|c|c|c|c|c|}
\hline Autor & $\begin{array}{l}\text { Nível de } \\
\text { evidência }\end{array}$ & Objetivo & Participantes & $\begin{array}{l}\text { Coleta de } \\
\text { dados }\end{array}$ & Resultados \\
\hline $\begin{array}{l}\text { Mulroy et al. } \\
\text { (2015) /EUA }\end{array}$ & III-2 & $\begin{array}{c}\text { Determinar } \\
\text { preditores de } \\
\text { dor nas } \\
\text { articulaçóes de } \\
\text { ombro em } \\
\text { pessoas com } \\
\text { paraplegia. }\end{array}$ & $\begin{array}{l} \\
\text { n= } 223 \\
\text { (198 homens e } \\
25 \text { mulheres); } \\
\text { Tempo médio } \\
\text { de lesão: } \\
9,3 \text { anos }( \pm 6,2) \text {; } \\
\text { Idade média: } \\
34,7 \text { anos } \\
\text { ( } \pm 9,3) ; \\
\text { Peso corporal } \\
\text { médio: } 74,1 \mathrm{~kg} \\
\quad( \pm 16,3)\end{array}$ & $\begin{array}{c}\text { Os participantes } \\
\text { foram avaliados } \\
\text { quanto à dor no } \\
\text { ombro durante } \\
3 \text { anos por meio } \\
\text { do WUSPI. } \\
\text { Foram } \\
\text { mensuradas } \\
\text { variáveis de } \\
\text { atividade diária } \\
\text { (velocidade } \\
\text { média e } \\
\text { distância de } \\
\text { propulsão; } \\
\text { transferência e } \\
\text { participaçáo } \\
\text { esportiva) e } \\
\text { variáveis de } \\
\text { torque de } \\
\text { ombro. }\end{array}$ & $\begin{array}{c}\text { Entre } \\
201 \text { participantes, } \\
39,8 \% \\
\text { desenvolveram } \\
\text { dor no ombro. } \\
\text { As variáveis de } \\
\text { atividade diária e } \\
\text { o torque } \\
\text { isométrico } \\
\text { máximo basal } \\
\text { foram menores } \\
\text { naqueles que } \\
\text { desenvolveram } \\
\text { dor no ombro. } \\
\text { O torque de } \\
\text { aduçáo do ombro } \\
\text { foi considerado } \\
\text { preditor de início } \\
\text { da dor. } \\
\text { No entanto, } \\
\text { nenhum dos } \\
\text { fatores foram } \\
\text { considerados } \\
\text { fortes preditores } \\
\text { de dor no ombro. }\end{array}$ \\
\hline $\begin{array}{c}\text { Gil-Agudo et al. } \\
\text { (2014) } \\
\text { /Espanha }\end{array}$ & IV & $\begin{array}{c}\text { Avaliar } \\
\text { alteraçóes no } \\
\text { ombro por meio } \\
\text { da } \\
\text { ultrassonografia } \\
\text { depois da } \\
\text { propulsão de } \\
\text { baixa e alta } \\
\text { intensidade. }\end{array}$ & $\begin{array}{c}\mathrm{n}=14 \text { homens; } \\
\text { Tempo médio } \\
\text { de lesão: } \\
7,51 \text { anos } \\
( \pm 4,56) ; \\
\text { Idade média: } \\
35,2 \text { anos } \\
( \pm 6,11) ; \\
\text { Peso corporal } \\
\text { médio: } 68,3 \mathrm{~kg} \\
( \pm 8,96)\end{array}$ & $\begin{array}{c}\text { Os participantes } \\
\text { de cada grupo } \\
\text { realizaram } \\
\text { propulsão na } \\
\text { CRM em alta } \\
\text { intensidade } \\
\text { sobre a esteira } \\
\text { motorizada. }\end{array}$ & $\begin{array}{c}\text { A magnitude de } \\
\text { forças e } \\
\text { momentos de } \\
\text { ombro } \\
\text { aumentou } \\
\text { durante a tarefa } \\
\text { de alta } \\
\text { intensidade; } \\
\text { O aumento das } \\
\text { forças de reaçáo } \\
\text { foi } \\
\text { correlacionado } \\
\text { com aumento } \\
\text { da espessura do } \\
\text { tendão do mm. } \\
\text { bíceps braquial e } \\
\text { com a } \\
\text { diminuiçáo do } \\
\text { espaço } \\
\text { subacromial; } \\
\text { Náo houve } \\
\text { alteraçóes por } \\
\text { meio da } \\
\text { ultrassonografia. }\end{array}$ \\
\hline
\end{tabular}


Tabela 1. Continuação...

\begin{tabular}{|c|c|c|c|c|c|}
\hline Autor & $\begin{array}{l}\text { Nível de } \\
\text { evidência }\end{array}$ & Objetivo & Participantes & $\begin{array}{l}\text { Coleta de } \\
\text { dados }\end{array}$ & Resultados \\
\hline $\begin{array}{c}\text { Gil- } \\
\text { Agudo et al. } \\
(2015) \\
\text { /Espanha }\end{array}$ & III-2 & $\begin{array}{c}\text { Comparar as } \\
\text { mudanças nas } \\
\text { forças e } \\
\text { momentos do } \\
\text { ombro e } \\
\text { alteraçóes } \\
\text { ultrassonográfic } \\
\text { as em usuários } \\
\text { de CRM e náo- } \\
\text { usuários de } \\
\text { CRM durante } \\
\text { uma atividade } \\
\text { de alta } \\
\text { intensidade. }\end{array}$ & $\begin{array}{c}\text { Grupo CRM } \\
\text { com LM: } \\
\text { n= } 22 \text { homens; } \\
\text { Tempo médio } \\
\text { de lesão: } \\
\text { 8,73 anos } \\
\text { ( } \pm 7,08) ; \\
\text { Idade média: } \\
35,5 \text { anos } \\
\text { ( } \pm 7,06) ; \\
\text { Peso corporal } \\
\text { médio: } \\
68,66 \text { kg } \\
\text { ( } \pm 10,76) ; \\
\text { Grupo sem } \\
\text { LM: } \\
\text { n= 12 homens; } \\
\text { Idade média: } \\
31,3 \text { anos } \\
\text { ( } \pm 7,46) ; \\
\text { Peso corporal } \\
\text { médio: } \\
73,87 \text { kg } \\
\text { ( } \pm 11,54)\end{array}$ & $\begin{array}{l}\text { Os } \\
\text { participantes de } \\
\text { cada grupo } \\
\text { realizaram } \\
\text { propulsáo em } \\
\text { alta intensidade } \\
\text { na esteira } \\
\text { motorizada. }\end{array}$ & $\begin{array}{c}\text { As forças e } \\
\text { momentos de } \\
\text { ombro dos } \\
\text { usuários de } \\
\text { CRM } \\
\text { aumentaram } \\
\text { em comparação } \\
\text { ao grupo } \\
\text { controle. } \\
\text { Não houve } \\
\text { alteraçóes por } \\
\text { meio da } \\
\text { ultrassonografia. }\end{array}$ \\
\hline $\begin{array}{l}\text { Russell et al. } \\
\text { (2015) /EUA }\end{array}$ & IV & $\begin{array}{l}\text { Determinar } \\
\text { como os } \\
\text { usuários de } \\
\text { CRM } \\
\text { modificam a } \\
\text { mecânica dos } \\
\text { MMSS } \\
\text { conforme o } \\
\text { aumento da } \\
\text { velocidade de } \\
\text { propulsão. }\end{array}$ & $\begin{array}{c}\mathrm{n}=40 \\
\text { (32 homens e } \\
8 \text { mulheres); } \\
\text { Tempo de } \\
\text { lesão: } \\
\text { 8,25 anos; } \\
\text { Idade média: } \\
35 \text { anos; } \\
\text { Peso corporal } \\
\text { médio: } 74,5 \mathrm{~kg} \\
\quad( \pm 18)\end{array}$ & $\begin{array}{l}\text { A cinemática } \\
\text { dos MMSS e as } \\
\text { forças de reação } \\
\text { geradas no aro } \\
\text { foram } \\
\text { mensuradas } \\
\text { durante a } \\
\text { propulsão de } \\
\text { velocidade livre } \\
\text { e rápida no } \\
\text { ergômetro } \\
\text { estacionário. }\end{array}$ & $\begin{array}{l}\text { O aumento da } \\
\text { velocidade da } \\
\text { propulsáo } \\
\text { promoveu } \\
\text { redução no } \\
\text { tempo de } \\
\text { contato com o } \\
\text { aro e aumentou } \\
\text { a magnitude } \\
\text { das forças de } \\
\text { reaçáo; } \\
\text { A magnitude } \\
\text { do momento } \\
\text { de ombro náo } \\
\text { corresponde, } \\
\text { necessariament } \\
\text { e, à elevação da } \\
\text { velocidade, mas } \\
\text { pode ser } \\
\text { alterada } \\
\text { conforme a } \\
\text { orientação dos } \\
\text { MMSS. }\end{array}$ \\
\hline
\end{tabular}


Tabela 1. Continuação...

\begin{tabular}{|c|c|c|c|c|c|}
\hline Autor & $\begin{array}{l}\text { Nível de } \\
\text { evidência }\end{array}$ & Objetivo & Participantes & $\begin{array}{c}\text { Coleta de } \\
\text { dados }\end{array}$ & Resultados \\
\hline $\begin{array}{l}\text { Rice et al. } \\
(2009) \text { /EUA }\end{array}$ & IV & $\begin{array}{c}\text { Examinar as } \\
\text { características } \\
\text { do impulso e } \\
\text { mudanças que } \\
\text { podem ocorrer } \\
\text { durante a } \\
\text { propulsão. }\end{array}$ & $\begin{array}{c}\mathrm{n}=21 \\
\text { (19 homens e } \\
2 \text { mulheres); } \\
\text { Tempo médio } \\
\text { de lesão: } \\
\text { 19,1 }( \pm 8,8) ; \\
\text { Idade média: } \\
44,8 \text { anos } \\
( \pm 9,6) ; \\
\text { Peso corporal } \\
\text { médio: } 79 \mathrm{~kg} \\
( \pm 14,96)\end{array}$ & $\begin{array}{l}\text { Dados cinéticos } \\
\text { foram } \\
\text { registrados } \\
\text { durante } \\
10 \mathrm{~min} . \mathrm{em} \\
\text { velocidade de } \\
1,4 \mathrm{~m} / \mathrm{s} .\end{array}$ & $\begin{array}{c}\text { A força de } \\
\text { reação máxima } \\
\text { diminuiu } \\
\text { conforme } \\
\text { aumentaram os } \\
\text { tempos de } \\
\text { impulsos e o } \\
\text { ciclo da } \\
\text { propulsão. }\end{array}$ \\
\hline
\end{tabular}

ACP = ângulo de contato posterior; $\mathrm{ADM}$ = amplitude de movimento; ALA = ângulo de liberaçáo anterior; $\mathrm{ARC}=$ arco; $\mathrm{CRM}=$ cadeira de rodas manual; $\mathrm{LM}=$ lesão medular; $\mathrm{MMSS}=$ membros superiores; SC = semicircular; SD = sem descrição; SLOP = single looping; WUSPI = Wheelchair User's Shoulder Pain Index. Fonte: Elaborada pelo autor.

\section{Discussão}

A presente revisão integrativa buscou identificar e avaliar fatores relacionados à eficiência da propulsão em CRM de usuários com paraplegia devido à LM. Sendo assim, foram identificados como fatores relacionados à eficiência da propulsão: os momentos não propulsivos; a velocidade dos MMSS na fase de recuperação; a posição da mão no período de liberação; o tamanho do encosto; a manutenção do peso corporal; o nível de atividade diária e a força de adutores de ombro; a intensidade da propulsão; a orientação dos MMSS; e o tempo de LM. Os achados foram categorizados da seguinte forma: ciclo e padróes de propulsão, configuraçóes da CRM, características dos usuários de CRM e dores e lesóes nos MMSS.

\subsection{Ciclo e padróes de propulsão}

Durante o ciclo da propulsão ocorre um intervalo em que a mão está em contato com o aro, gerando força suficiente para realizar propulsão da CRM, entre dois instantes em que a mão permanece em contato com o aro, mas não gera força suficiente para realizar propulsão (Kwarciak et al., 2009a).

Desta forma, o ciclo da propulsão é divido em: fase de contato e fase de recuperaçáo. A fase de contato é subdividida em 3 períodos: contato inicial, propulsão e liberação (Figura 2). O contato inicial é compreendido pelo contato da mão no aro, mas sem gerar força suficiente para produzir propulsão da CRM. Esse período é caracterizado como o momento não propulsivo do contato da mão no aro. No período de propulsão, a mão ainda permanece em contato com o aro, mas gerando força suficiente para produzir propulsão da CRM. Esse período é definido como o momento propulsivo. Assim como a fase de contato, a fase de liberação é definida como o momento não propulsivo do contato da mão no aro. Neste período, a mão está sendo liberada do aro e as forças estão sendo cessadas completamente. Finalizando o ciclo da propulsão, na fase de recuperação, em contraste com 
a fase de contato, configura-se um instante em que a mão não entra em contato com o aro, ou seja, não há forças agindo para propulsionar a CRM (Kwarciak et al., 2009a).

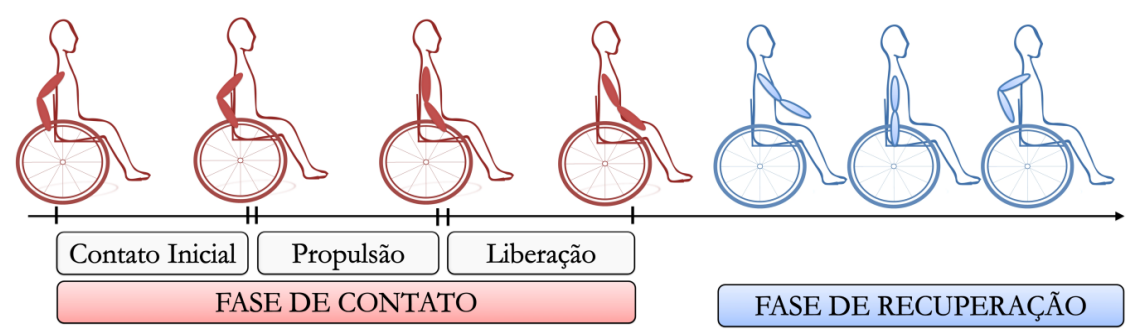

Figura 2. Ciclo da propulsão. Fonte: Elaborada pelo autor.

Nos períodos de contato inicial e de liberação ocorrem momentos de frenagem (ato de frear) causados por um momento não propulsivo entre a mão e o aro de propulsão (Kwarciak et al., 2009a). De acordo com os resultados do estudo, nas velocidades de $1,08 \mathrm{~m} / \mathrm{s}$ e $1,74 \mathrm{~m} / \mathrm{s}, 70 \%$ e $88 \%$ de todos os impulsos do período de contato inicial foram identificados como momentos de frenagem, respectivamente (Kwarciak et al., 2009a). Do mesmo modo, em ambas as velocidades, durante o período de liberação, $43 \%$ e $63 \%$ de todos os impulsos foram identificados como momentos de frenagem, respectivamente. Esses dados demonstram que a perda de potência e o aumento do momento de frenagem ocorrem conforme a velocidade do impulso aumenta durante os períodos de contato inicial e de liberação. Logo, os profissionais, ao realizarem treinos de propulsão, precisam considerar a magnitude da velocidade nos períodos de momento não propulsivo da mão (Kwarciak et al., 2009a).

O tipo de padrão de propulsão utilizado pelo usuário pode alterar o momento de frenagem e a perda de potência (Kwarciak et al., 2009a). O padrão de propulsão no qual a mão fica abaixo do aro durante a fase de recuperação apresenta menor magnitude do momento de frenagem e perda de potência durante o período de contato inicial em relação ao padrão de propulsão com a mão posicionada acima do aro. A aplicação de força durante o ciclo da propulsão pode ser prejudicada pela diminuição dos momentos de frenagem e perda de potência no período de contato inicial (Kwarciak et al., 2009a). Desta forma, os usuários de CRM devem ser encorajados a utilizar o padrão de propulsão no qual as mãos fiquem abaixo do aro durante a fase de recuperação.

Diversos estudos descrevem quatro padróes de propulsão realizados por usuários de CRM: semicircular; single looping (SLOP); double looping (DLOP) e arco. No padrão semicircular, por um lado, as mãos criam uma trajetória abaixo do aro durante a fase de recuperação. Por outro lado, no padrão SLOP a trajetória da mão forma um único looping acima do aro. No padrão DLOP, após a fase de contato, as mãos se elevam e, posteriormente, caem, formando dois looping, finalizando o ciclo de propulsão (Shimada et al., 1998). E, por fim, no padrão arco, durante a fase de recuperação a mão retorna ao aro pelo mesmo trajeto realizado na fase de contato (Boninger et al., 2002) (Figura 3). 


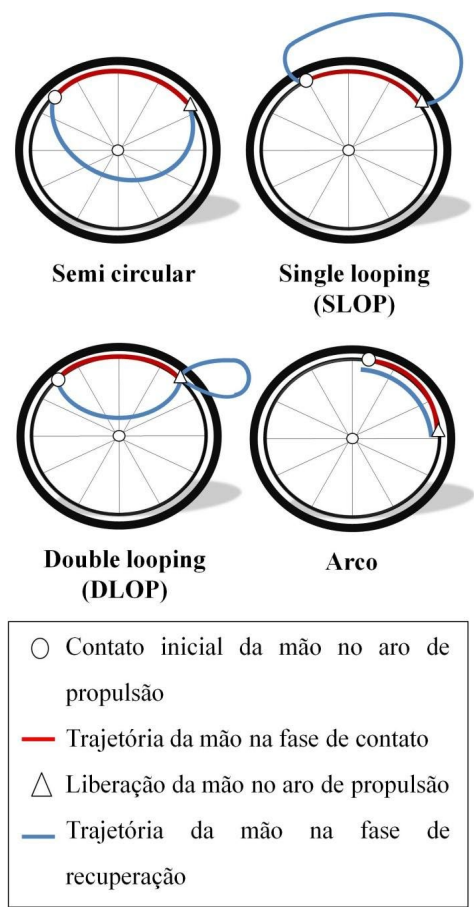

Figura 3. Padrões de propulsão. Fonte: Elaborada pelo autor.

O padrão SLOP é utilizado preferencialmente pelos usuários de CRM em relação a outros padróes de propulsão (Raina et al., 2012). Esses dados corroboram os resultados encontrados por Boninger et al. (2002), visto que os participantes com paraplegia também utilizaram, preferencialmente, o padrão SLOP. Entretanto, o padrão semicircular é o mais eficiente dentre os padróes de propulsão, uma vez que o usuário realiza menos movimentos para impulsionar a CRM (Boninger et al., 2002). É importante ressaltar que no instante em que a mão do usuário entra em contato com o aro para iniciar a fase de contato são geradas diversas forças de reação direcionadas aos MMSS. Com base nessa perspectiva, o padrão semicircular propicia menos impulsos no aro, e, consequentemente, menos forças são direcionadas aos MMSS. Em comparação, o padrão arco é considerado o menos eficiente, visto que o usuário precisará impulsionar inúmeras vezes para atingir a mesma distância, aumentando os riscos de dores e lesóes nos MMSS por conta do excesso de movimentos dos MMSS (Boninger et al., 2002).

Durante a propulsão, diversas forças direcionais são geradas no instante em que a mão entra em contato com o aro de propulsão: Fx (força anterior/posterior), Fy (força superior/inferior) e Fz (força medial/lateral) (Cooper, 2009) (Figura 4). A velocidade da mão antes do contato com o aro de propulsão na fase de recuperação está correlacionada com a força de reação na direção tangencial (entre a Fx anterior e a Fz lateral), também chamada de força efetiva (Raina et al., 2012). O aumento da magnitude da Fx (tangencial) de usuários com paraplegia durante a fase de contato está associado ao aumento da velocidade dos MMSS na fase de recuperação em relação aos participantes com tetraplegia. No entanto, não foi encontrada correlação entre a força de reação e os padróes de propulsão (Raina et al., 2012). 


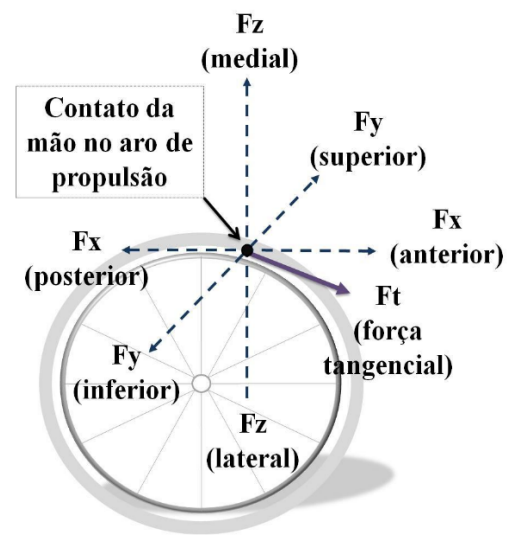

Figura 4. Forças de reação durante a propulsão em cadeira de rodas manual.

Fonte: Elaborada pelo autor.

Esses dados indicam que a velocidade dos MMSS é um fator que deve ser levado em consideração durante o percurso em que a mão entra em contato novamente com o aro. Entretanto, quando forças são aplicadas em outras direçôes durante a propulsão, como na direção anterior, por exemplo, consequentemente, são geradas forças de reação na direção posterior, causando impacto sobre as estruturas dos MMSS. Desta forma, é imprescindível que os profissionais compreendam a percepção do usuário quanto à exigência dos MMSS associada à magnitude da velocidade durante a fase de recuperação.

$\mathrm{O}$ aumento do ângulo de contato promove uma propulsão com movimentos longos e suaves que maximizam o tempo de contato e diminuem as forças de reação aplicadas ao aro, minimizando a carga e as forças de reação sobre o ombro (Boninger et al., 2000). Contudo, é essencial compreender qual a localização da mão no aro nos períodos de contato inicial e de liberaçáo. Tanto o ângulo de contato inicial mais posterior (ACP) quanto o ângulo de liberaçáo mais anterior (ALA) da mão estão associados a uma cadência reduzida e com aumento da distância entre os inícios de cada ciclo de propulsão (Requejo et al., 2015). Ademais, o ACP demonstra aumentar a demanda dos mm. flexores do ombro, enquanto o ALA aumenta a demanda dos $\mathrm{mm}$. adutores e rotadores internos (Requejo et al., 2015).

O aumento de forças e momentos articulares de abdução e rotação interna de ombro pode favorecer o surgimento de espessamento adaptativo do ligamento coracoacromial e dores na região do ombro (Mercer et al., 2006). De acordo com os dados de Requejo et al. (2015), essa condição está associada ao ALA. Sendo assim, a fim de maximizar o ângulo de propulsão, é indicado que os usuários realizem um contato inicial mais posterior ao aro de propulsão em vez de realizar um contato da mão mais anteriormente.

Segundo a Organização Mundial da Saúde (2012), os serviços de dispensação de cadeira de rodas devem seguir 8 etapas sequenciais: 1) Encaminhamento e agendamento; 2) Avaliação; 3) Prescrição e seleção; 4) Financiamento e aquisição; 5) Preparação do produto; 6) Adequação; 7) Treinamento de usuários; e 8) Manutenção, reparos e acompanhamento. As evidências apresentadas e discutidas sobre ciclo e padróes de propulsão podem ser traduzidas na prática clínica na etapa do treinamento de usuários. Assim, baseando-se nos fatores ciclo e padróes de propulsão, compete aos terapeutas ocupacionais da prática e dos serviços de adequação postural conduzirem treinos de 
propulsão, com o propósito de maximizar a função dos MMSS e, consequentemente, promover mobilidade funcional nas AVD's.

Usuários de CRM comumente relatam dores nos MMSS, sendo o ombro a região mais afetada, seguido de punho e mão (Cooper et al., 1998). Estudos demonstram que os usuários de CRM têm alto risco de lesão no arco coracoacromial (Mercer et al., 2006) e no nervo mediano (Boninger et al., 1999). Os padrões de propulsão caracterizados por movimentos repetitivos, como o padrão SLOP, por exemplo, é uma das principais causas. Os movimentos do padrão SLOP apresentam ser a forma mais espontânea de propulsionar a CRM pelos usuários (Boninger et al., 1999). Desta maneira, os terapeutas precisam ter em vista que alterar o padrão de propulsão com movimentos adequados é um processo que demanda tempo, uma vez que determinados movimentos se tornam hábitos por serem realizados repetitivamente no cotidiano do usuário.

\subsection{Configuraçóes da cadeira de rodas manual}

O uso do encosto baixo (regulada em $50 \%$ do comprimento do tronco) pode proporcionar tempos de impulso mais longos, cadência reduzida e aumento dos ângulos do ciclo de propulsão (Yang et al., 2012). O contato inicial da mão ao aro mais posteriormente e uma recuperação da mão mais adiante resulta em maiores ângulos de impulso durante o ciclo da propulsão (Yang et al., 2012). Em relação ao encosto com $3^{\circ}$ de inclinação, demonstrou favorecer o aumento da cadência e o deslocamento do contato inicial e da recuperação da mão para frente durante a fase de contato (Yang et al., 2012). Essa condição proporciona maior amplitude de movimento de flexão/extensão de ombro, bem como aumento de forças e momentos (Yang et al., 2012).

A utilização de um encosto baixo demonstrou ser benéfica quanto à diminuição de esforços realizados pelos MMSS durante a propulsão em CRM. Entretanto, a utilização do encosto baixo pode causar mais instabilidade e desconforto por conta da limitação do apoio dorsal (Medola et al., 2014). Sendo assim, na seleção do tamanho do encosto durante a prescrição da CRM os profissionais também devem considerar a percepção do usuário quanto ao conforto e estabilidade posterior.

Estudos prévios demonstraram que outros componentes das CRM e sua configuração de forma inadequada podem exigir intensamente os MMSS, provocar diminuição da mobilidade e instabilidade da CRM do usuário (Medola et al., 2014). Assentos posicionados muito acima em relação às rodas traseiras promovem aumento na frequência de impulso, elevando o risco de lesóes nos MMSS por esforços repetitivos (Boninger et al., 2000). Além disso, evidências demonstram que assentos posicionados em ângulo reto podem provocar maiores riscos de dores e lesôes no ombro em usuários com paraplegia em comparação ao uso de assentos posicionados em ângulo agudo (Giner-Pascual et al., 2011). Para promover eficiência da propulsão e menor gasto de energia, é recomendado que o assento esteja posicionado a uma altura em que o ângulo do cotovelo, no momento do contato da mão ao aro de propulsão, varie de $100^{\circ}$ a $120^{\circ}$ (Van Der Woude et al., 1989).

Do mesmo modo que o assento, o ajuste da posição das rodas influencia a eficiência da propulsão. A menor distância vertical entre o eixo da roda e o ombro, e o avanço na posição do eixo horizontal proporcionam aumento do ângulo de impulso, enquanto diminuem a frequência dos ciclos da propulsão e o atrito de rolamento (Boninger et al., 
2000). Em outras palavras, as rodas traseiras posicionadas em uma altura que proporcione maior contato da mão ao aro de propulsão (eixo X), assim como posicionadas de forma mais avançada (eixo Y), podem promover a eficiência da biomecânica da propulsão devido à diminuição de impulsos frequentes e de forças de reação opostas que dificultam a propulsão (Figura 5). Apesar desses benefícios, a posição das rodas traseiras mais a frente causam instabilidade, aumentando o risco de virar a CRM para trás com mais facilidade. Ao indicar ajustes na posição das rodas traseiras, é fundamental considerar o tempo de lesão e a percepção do usuário em relação à estabilidade.

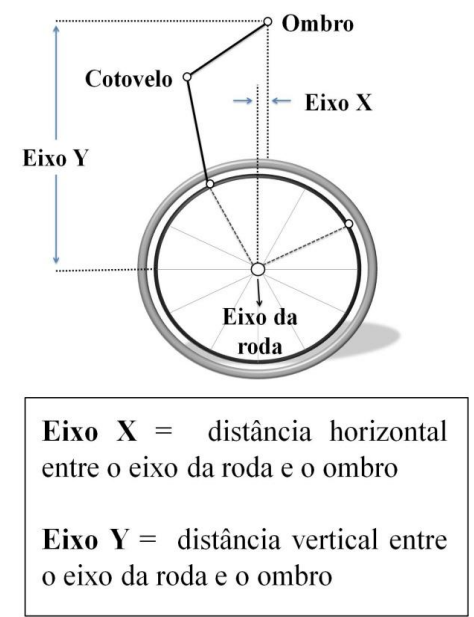

Figura 5. Posição dos eixos. Fonte: Elaborada pelo autor.

O tipo de material que constitui a estrutura da CRM, bem como o tipo de pneu, também pode afetar a eficiência da biomecânica da propulsão (Medola et al., 2014). Estudos indicam que os usuários devem utilizar, preferencialmente, uma CRM ultralight, a qual é constituída por titanium e fibra de carbono (Medola et al., 2014). Por um lado, esse tipo de CRM diminui os impactos sobre as estruturas dos MMSS, em razão de que são mais leves e resistentes, produzindo menor resistência ao rolamento, isto é, diminuindo as forças que se opõem ao propulsionar a CRM (Cooper et al., 1997). Por outro lado, as CRM constituídas por alumínio são mais pesadas e, consequentemente, exigem mais dos MMSS durante a propulsão (Cooper et al., 1997). Os pneus do tipo pneumáticos também contribuem para produzir menor resistência ao rolamento em comparação aos pneus do tipo sólidos (Kwarciak et al., 2009b). Assim, pneus pneumáticos contribuem para diminuir o risco de dores e lesóes por esforços repetitivos, devido à redução da frequência de impulsos e das forças de reação geradas aos MMSS.

O processo de configurar a cadeira de rodas, também chamado de etapa de adequaçáo, é realizado pelos serviços de dispensação de CRM. Essa etapa é fundamental para conceder aos usuários um ciclo de propulsão seguro e confortável, diminuindo riscos de quedas e dores e lesões nos MMSS (Organização Mundial da Saúde, 2012). Assim sendo, o terapeuta ocupacional realiza adequação da postura e confere o tamanho, o tipo de material e ajustes dos componentes da CRM, de acordo com as necessidades do usuário. 
As evidências demostraram que o tamanho do encosto é um fator relacionado à eficiência da propulsão. Além disso, outros fatores podem influenciar a propulsão, como a posição das rodas traseiras em relação ao ombro do usuário e a seleção do tipo de material que constitui a CRM. Apesar das evidências apresentadas e discutidas no artigo, é importante ressaltar que é essencial considerar a opinião e as necessidades dos usuários ao configurar a cadeira de rodas. $\mathrm{O}$ usuário, ao utilizar um encosto baixo e as rodas traseiras muito à frente, poderá sentir desconforto e fadiga, devido à redução de apoio para as costas, além de instabilidade durante a propulsão, aumentando o risco de quedas. Ademais, a CRM ultralight, o dispositivo mais resistente e leve do mercado, não está disponível para ser dispensada pelos serviços públicos de saúde. Portanto, é necessário que os profissionais realizem a configuração dos componentes da CRM para promover propulsão com eficiência, considerando a opiniâo dos usuários e familiares/cuidadores sobre esta.

\subsection{Características do usuário de cadeira de rodas manual}

O peso corporal é um fator que pode aumentar as forças e momentos de ombro durante a propulsão (Collinger et al., 2008). As forças e momentos de ombro aumentam conforme o aumento da velocidade da propulsão. $\mathrm{Na}$ fase de contato, a Fx posterior é o maior componente de força direcional, enquanto o momento de rotaçáo interna é o maior componente de momento direcional (Collinger et al., 2008). A força articular máxima ocorre perto do início da fase de contato, enquanto o ombro é estendido, abduzido e rotacionado internamente (Collinger et al., 2008)

$\mathrm{O}$ estado de sobrepeso pode promover aumento da resistência ao rolamento, sendo necessário gerar mais força durante a propulsão. Desta forma, as estruturas dos MMSS são altamente exigidas por conta da frequência constante de impulsos e, consequentemente, os usuários ficam mais suscetíveis a lesōes no nervo mediano e ao desenvolvimento de compressóes, como a síndrome de túnel do carpo (Boninger et al., 1999).

Assim como o peso corporal, a estatura do usuário também influencia a eficiência da propulsão. A distância vertical entre o eixo da CRM e o ombro pode diminuir ou aumentar de acordo com a estatura, ou seja, quanto mais alto for o usuário, mais distante do aro de propulsão estará, e, portanto, menor será o ciclo propulsivo (Boninger et al., 2000). Desta maneira, os profissionais devem ajustar os eixos (horizontal e vertical) e o assento da CRM apropriadamente com a altura e o comprimento do braço do usuário, aproximando-o ao aro de propulsão.

Durante a avaliação física, o terapeuta ocupacional realiza a mensuração antropométrica para selecionar a cadeira de rodas disponível no mercado de acordo com as medidas do usuário (Organização Mundial da Saúde, 2012). Para tal, são necessários um ou dois profissionais treinados e com conhecimento de como e quais locais do corpo devem ser mensurados (Organização Mundial da Saúde, 2012). Além disso, é imprescindível o usuário provar a CRM para não correr o risco de adquiri-la com o tamanho que não esteja de acordo com as medidas do seu corpo. O uso de uma CRM de tamanho inadequado não apenas pode afetar a eficiência da propulsão, mas também poderá aumentar o risco de gerar úlceras/feridas por pressão e até mesmo acarretar o abandono do dispositivo pelo usuário. 


\subsection{Dores e lesóes nos membros superiores}

O nível de atividade diária demonstrou ser um fator relacionado a dores no ombro. De acordo com o estudo de Mulroy et al. (2015), os participantes que desenvolveram dor no ombro demonstraram menor nível de velocidade diária $(2,4 \mathrm{~km} / \mathrm{h})$, em relação aos participantes que permaneceram sem dor $(2,8 \mathrm{~km} / \mathrm{h})$.

Além disso, constatou-se que o momento de adução de ombro está relacionado inicialmente como preditor de início da dor, no entanto, representou apenas $7,5 \%$ do início da dor no ombro. Apesar de não terem sido encontrados fortes preditores, houve correlação das dores nos ombros com a diminuição das atividades diárias e de força da musculatura adutora do ombro em paraplégicos (Mulroy et al., 2015).

O aumento nas forças e momentos de ombro em todas as direçóes está relacionado à propulsão tardia durante uma tarefa considerada de alta intensidade (Gil-Agudo et al., 2014). Ademais, pode favorecer aumento na espessura do tendão da cabeça longa do mm. bíceps braquial e diminuição do espaço subacromial (Gil-Agudo et al., 2014). Esses resultados evidenciaram que uma propulsão em alta velocidade exige mais esforço dos MMSS, ampliando a predisposição ao desenvolvimento de dores e lesóes (Gil-Agudo et al., 2014).

Impactos sobre o ombro também podem ocorrer na propulsão precoce durante a atividade de alta intensidade (Gil-Agudo et al., 2015). Evidenciou-se que os momentos de ombro aumentam em todas as direçóes, exceto para Fx lateral, bem como os momentos de abdução e extensão de ombro. Além disso, dores no ombro podem estar associadas ao aumento do tendão da cabeça longa do $\mathrm{mm}$. bíceps braquial (Gil-Agudo et al., 2015).

Os posicionamentos dos MMSS levam a diferentes magnitudes de momentos de ombro na mesma condição de propulsão (Russell et al., 2015). Evidencia-se também que a magnitude do momento de ombro depende das forças geradas sobre o centro de massa (CM) do antebraço e braço (Russell et al., 2015). De acordo com o autor, durante a propulsão de $1,72 \mathrm{~m} / \mathrm{s}$, em que a força de reação $(60 \mathrm{~N})$ foi orientada anteriormente ao CM do antebraço e braço associada ao momento extensor de cotovelo, ocorreu redução da magnitude do momento de ombro $(13 \mathrm{Nm})$. Em contrapartida, na condição de propulsão de $1,82 \mathrm{~m} / \mathrm{s}$, em que a força de reação $(54 \mathrm{~N})$ foi orientada posteriormente ao CM do antebraço e braço associada ao momento flexor de cotovelo, ocorreu aumento da magnitude do momento de ombro (27 Nm) (Russell et al., 2015). Os dois exemplos indicam que a magnitude do momento de ombro náo corresponde necessariamente à velocidade da propulsão, mas pode ser reduzida ou elevada por meio de momentos de flexão ou extensão de cotovelo associados às forças de reação na direção anterior e posterior sobre o CM do antebraço e braço.

Gil-Agudo et al. $(2014,2015)$ apresentaram resultados semelhantes a estudos prévios (Mercer et al., 2006; Collinger et al., 2008) com associaçôes de maior tendência ao surgimento de dores e lesóes com aumento de forças e momentos de ombro, após os participantes realizarem propulsão rápida. Russell et al. (2015) ainda demonstraram que a orientação das forças de reação associadas aos momentos de cotovelo em relação ao $\mathrm{CM}$ do antebraço e braço é essencial para diminuir as forças dirigidas ao ombro. Durante a propulsão em alta velocidade, a reorientação das forças de reação, geradas pela posição 
dos MMSS em relação aos CM do antebraço e braço, demonstrou atenuar as forças direcionadas ao ombro.

Em contrapartida aos estudos anteriores, Rice et al. (2009) evidenciaram que os participantes que realizaram uma tarefa longa e em alta velocidade $(1,4 \mathrm{~m} / \mathrm{s})$ ficaram mais tempo na fase de contato, enquanto as forças de reação diminuíram durante a propulsão e também não demonstraram ineficiência da propulsão. Verificou-se que a experiência dos usuários (média de 20 anos de lesão) pode ser um fator que contribui para adaptaçóes na biomecânica do ciclo da propulsão, que, por sua vez, diminui a exigência de movimentos intensos dos MMSS, favorecendo a eficiência da propulsão durante a tarefa.

A propulsão em CRM exige a movimentação dos MMSS para promover mobilidade funcional. Usuários de CRM com LM comumente relatam dores nos ombros que afetam a função do MMSS durante a propulsão (Requejo et al., 2008). As evidências demonstram que fatores como atividades intensas, as quais exigem movimentos repetitivos, e determinados padróes de movimentos durante o ciclo da propulsáo podem aumentar o risco de dores e lesóes nos ombros e, assim, prejudicar a eficiência da propulsão. Desta forma, concerne aos terapeutas ocupacionais orientar quanto aos movimentos adequados durante o ciclo da propulsão, a fim de prevenir os riscos de dores e lesões nos ombros. Ademais, é necessário ressaltar que a postura do usuário e a configuração dos componentes da CRM também são fatores fundamentais para prevenir a função dos MMSS. Logo, todos os fatores citados devem ser levados em consideração em conjunto para realizar a propulsão em CRM com maior eficiência.

\section{Conclusáo}

A presente revisão integrativa da literatura forneceu evidências sobre fatores relacionados à eficiência da propulsão de indivíduos com paraplegia devido à LM. As evidências indicam que fatores como o ciclo e padrões de propulsão, configuraçóes de CRM, características do usuário e dores e lesôes nos MMSS estão relacionados à eficiência da propulsão em CRM de usuários com paraplegia devido à LM.

Apesar dos estudos incluídos nesta revisão apresentarem importantes contribuições para a prática clínica, a maioria utilizou o desenho de estudo série de casos, configurando-os como nível IV de evidência. Sendo assim, sugere-se que as futuras investigaçóes sejam realizadas com outros tipos de desenho de estudo, como estudos longitudinais e comparativos, por exemplo, a fim de produzir conhecimento científico com maiores níveis de evidência.

\section{Referências}

Boninger, M. L., Baldwin, M., Cooper, R. A., Koontz, A., \& Chan, L. (2000). Manual wheelchair pushrim biomechanics and axle position. Archives of Physical Medicine and Rehabilitation, 81(5), 608-613. PMid:10807100. http://dx.doi.org/10.1016/S0003-9993(00)90043-1.

Boninger, M. L., Cooper, R. A., Baldwin, M. A., Shimada, S. D., \& Koontz, A. (1999). Wheelchair pushrim kinetics: body weight and median nerve function. Archives of Physical Medicine and Rehabilitation, 80(8), 910915. PMid:10453767. http://dx.doi.org/10.1016/S0003-9993(99)90082-5. 
Boninger, M. L., Souza, A. L., Cooper, R. A., Fitzgerald, S. G., Koontz, A. M., \& Fay, B. T. (2002). Propulsion patterns and pushrim biomechanics in manual wheelchair propulsion. Archives of Physical Medicine and Rehabilitation, 83(5), 718-723. PMid:11994814. http://dx.doi.org/10.1053/apmr.2002.32455.

Brasil. Conselho Federal de Fisioterapia e Terapia Ocupacional - COFFITO. (2015, 20 de novembro). Resoluçáo no 458, de 20 de novembro de 2015. Dispóe sobre o uso da Tecnologia Assistiva pelo terapeuta ocupacional e dá outras providências. Diário Oficial [da] República Federativa do Brasil, Brasília. Recuperado em 10 de janeiro de 2020, de https://www.coffito.gov.br/nsite/?p=322

Chow, J. W., \& Levy, C. E. (2011). Wheelchair propulsion biomechanics and wheelers' quality of life: an exploratory review. Disability and Rehabilitation. Assistive Technology, 6(5), 365-377. PMid:20932232. http://dx.doi.org/10.3109/17483107.2010.525290.

Collinger, J. L., Boninger, M. L., Koontz, A. M., Price, R., Sisto, S. A., Tolerico, M. L., \& Cooper, R. A. (2008). Shoulder biomechanics during the push phase of wheelchair propulsion: a multisite study of persons with paraplegia. Archives of Physical Medicine and Rehabilitation, 89(4), 667-676. PMid:18373997. http://dx.doi.org/10.1016/j.apmr.2007.09.052.

Cooper, R. A. (2009). SMARTWheel: from concept to clinical practice. Prosthetics and Orthotics International, 33(3), 198-209. PMid:19658010. http://dx.doi.org/10.1080/03093640903082126.

Cooper, R. A., Boninger, M. L., Robertson, R., \& Heavy, H. (1998). Heavy Handed: repetitive strain injury among manual wheelchair users. Team Rehab Report, 9(2), 35-38. Recuperado em 20 de novembro de 2018, de http://rotamobility.com/docs/cooper.pdf

Cooper, R. A., Gonzalez, J., Lawrence, B., Renschler, A., Boninger, M. L., \& Vansickle, D. P. (1997). Performance of selected lightweight wheelchairs on ANSI/RESNA tests. Archives of Physical Medicine and Rehabilitation, 78(10), 1138-1144. PMid:9339166. http://dx.doi.org/10.1016/S0003-9993(97)90141-6.

DiGiovine, C., Koontz, A., \& Boninger, M. (2006). Advances in manual wheelchair technology. Topics in Spinal Cord Injury Rehabilitation, 11(4), 1-14. http://dx.doi.org/10.1310/67TW-L3UD-RUYG-7UKJ.

Gil-Agudo, A., Mozos, M. S., Ruiz, B. C., Del-Ama, A. J., Pérez-Rizo, E., Segura-Fragoso, A., \& Jiménez-Díaz, F. (2015). Shoulder kinetics and ultrasonography changes after performing a high-intensity task in spinal cord injury subjects and healthy controls. Spinal Cord, 54(4), 277-282. PMid:26282495.

http://dx.doi.org/10.1038/sc.2015.140.

Gil-Agudo, Á., Solís-Mozos, M., Crespo-Ruiz, B., Del-Ama Eng, A. J., Pérez-Rizo, E., Segura-Fragoso, A., \& Jiménez-Díaz, F. (2014). Echographic and kinetic changes in the shoulder joint after manual wheelchair propulsion under two different workload settings. Frontiers in Bioengineering and Biotechnology, 2, 77. PMid:25566539. http://dx.doi.org/10.3389/fbioe.2014.00077.

Giner-Pascual, M., Alcanyis-Alberola, H., Millan-González, G., Aguilar-Rodriguez, M., \& Querol, F. (2011). Shoulder pain in cases of spinal injury: influence of the position of the wheelchair seat. International Journal of Rehabilitation Research, 34(4), 282-289. PMid:21971486. http://dx.doi.org/10.1097/MRR.0b013e32834a8fd9.

Kwarciak, A. M., Sisto, S. A., Yarossi, M., Price, R., Komaroff, E., \& Boninger, M. L. (2009a). Redefining the manual wheelchair stroke cycle: identification and impact of nonpropulsive pushrim contact. Archives of Physical Medicine and Rehabilitation, 90(1), 20-26. PMid:19154825. http://dx.doi.org/10.1016/j.apmr.2008.07.013.

Kwarciak, A. M., Yarossi, M., Ramanujam, A., Dyson-Hudson, T. A., \& Sisto, S. A. (2009b). Evaluation of wheelchair tire rolling resistance using dynamometer-based coast-down tests. Journal of Rehabilitation Research and Development, 46(7), 931-938. PMid:20104415. http://dx.doi.org/10.1682/JRRD.2008.10.0137.

Masini, M. (2001). Estimativa da incidência e prevalência de lesão medular no Brasil. J Bras Neurocirurg, 12(2), $97-$ 100.

Medola, F. O., Elui, V. M. C., Santana, C. S., \& Fortulan, C. A. (2014). Aspects of manual wheelchair configuration affecting mobility: a review. Journal of Physical Therapy Science, 26(2), 313-318. PMid:24648656. http://dx.doi.org/10.1589/jpts.26.313.

Mercer, J. L., Boninger, M., Koontz, A., Ren, D., Dysonhudson, T., \& Cooper, R. (2006). Shoulder joint kinetics and pathology in manual wheelchair users. Clinical Biomechanics, 21(8), 781-789. PMid:16808992. http://dx.doi.org/10.1016/j.clinbiomech.2006.04.010.

Mulroy, S. J., Hatchett, P., Eberly, V. J., Haubert, L. L., Conners, S., \& Requejo, P. S. (2015). Shoulder strength and physical activity predictors of shoulder pain in people with paraplegia from spinal injury: prospective 
cohort study. Physical Therapy, 95(7), 1027-1038. PMid:25721123.

http://dx.doi.org/10.2522/ptj.20130606.

National Health and Medical Research Council - NHMRC. (1998). A guide to the development, implementation and evaluation of clinical practice guidelines. Recuperado em 18 de novembro de 2018, de https://www.health.qld.gov.au/_data/assets/pdf_file/0029/143696/nhmrc_clinprgde.pdf

Organização Mundial da Saúde - OMS. (2012). Pacote de treinamento em serviços para cadeiras de rodas. Recuperado em 15 de janeiro de 2020 , de https://apps.who.int/iris/bitstream/handle/10665/78236/9789241503471_reference_manual_por.pdf?sequen ce $=48$

Polia, A. A., \& Castro, D. H. (2007). A lesão medular e suas sequelas de acordo com o modelo de ocupação humana. Cadernos de Terapia Ocupacional da UFSCar, 15(1), 19-29.

Raina, S., Mcnitt-Gray, J., Mulroy, S., \& Requejo, P. (2012). Effect of choice of recovery patterns on handrim kinetics in manual wheelchair users with paraplegia and tetraplegia. The Journal of Spinal Cord Medicine, 35(3), 148-155. PMid:22507024. http://dx.doi.org/10.1179/2045772312Y.0000000013.

Requejo, P., Mulroy, S., Haubert, L. L., Newsam, C., Gronley, J. A., \& Perry, J. (2008). Evidence-Based strategies to preserve shoulder function in manual wheelchair users with spinal cord injury. Topics in Spinal Cord Injury Rehabilitation, 13(4), 86-119. http://dx.doi.org/10.1310/sci1304-86.

Requejo, P. S., Mulroy, S. J., Ruparel, P., Hatchett, P. E., Haubert, L. L., Eberly, V. J., \& Gronley, J. K. (2015). Relationship between hand contact angle and shoulder loading during manual wheelchair propulsion by individuals with paraplegia. Topics in Spinal Cord Injury Rehabilitation, 21(4), 313-324. PMid:26689696. http://dx.doi.org/10.1310/sci2104-313.

Rice, I., Impink, B., Niyonkuru, C., \& Boninger, M. (2009). Manual wheelchair stroke characteristics during an extended period of propulsion. Spinal Cord, 47(5), 413-417. PMid:19002155. http://dx.doi.org/10.1038/sc.2008.139.

Russell, I. M., Raina, S., Requejo, P. S., Wilcox, R. R., Mulroy, S., \& McNitt-Gray, J. L. (2015). Modifications in wheelchair propulsion technique with speed. Frontiers in Bioengineering and Biotechnology, 3(171), 171. PMid:26579513. http://dx.doi.org/10.3389/fbioe.2015.00171.

Shimada, S. D., Robertson, R. N., Bonninger, M. L., \& Cooper, R. A. (1998). Kinematic characterization of wheelchair propulsion. Journal of Rehabilitation Research and Development, 35(2), 210-218. PMid:9651893.

Souza, M. T., Silva, M. D., \& Carvalho, R. (2010). Revisão integrativa: o que é e como fazer. Einstein, 8(1), 102106. PMid:26761761. http://dx.doi.org/10.1590/s1679-45082010rw1134.

Van Der Woude, L. H. V., Veeger, D. J., Rozendal, R. H., \& Sargeant, T. J. (1989). Seat height in handrim wheelchair propulsion. Journal of Rehabilitation Research and Development, 26(4), 31-50. PMid:2600867.

World Health Organization - WHO. (2013). Spinal cord injury: key facts. Recuperado em 20 de outubro de 2018, de http://www.who.int/news-room/fact-sheets/detail/spinal-cord-injury

Yang, Y. S., Koontz, A. M., Yeh, S. J., \& Chang, J. J. (2012). Effect of backrest height on wheelchair propulsion biomechanics for level and uphill conditions. Archives of Physical Medicine and Rehabilitation, 93(4), 654-659. PMid:22325682. http://dx.doi.org/10.1016/j.apmr.2011.10.023.

\section{Contribuiçáo dos Autores}

Haidar Tafner Curi: organização, leitura, análise e discussão das fontes bibliográficas; concepção das imagens; construção e revisão crítica do texto. Jaqueline de Lima: construção e revisão crítica do texto. Eliana Chaves Ferretti: organização, leitura, análise e discussão das fontes bibliográficas; revisão do texto e orientação da pesquisa. Todos os autores aprovaram a versão final do texto.

\section{Autor para correspondência}

Eliana Chaves Ferretti

e-mail: chavesferretti@gmail.com 